\title{
Microscopy Study of the Morphology of Carbonized ZIF-67 Tailored by CTAB
}

\author{
Changqian Feng ${ }^{1}$, Jing Sui ${ }^{1}$, Yue $\mathrm{Xia}^{1}$, Jianhua $\mathrm{Yu}^{1}$, Liyan $\mathrm{Yu}^{1}$, Lifeng Dong ${ }^{1,2}$ \\ 1. College of Materials Science and Engineering, Qingdao University of Science and Technology, \\ Qingdao 266042, China \\ 2. Department of Physics, Hamline University, St. Paul 55104, USA
}

Metal-organic frameworks (MOFs) have been widely used in gas storage, catalysis, biomedical and electrochemical studies [1]. Among them, the carbonized ZIF-67 exhibits excellent performance in catalytic oxygen reduction reactions (ORR) [2], and the addition of cetyl trimethyl ammonium bromide (CTAB) has a great influence on its ORR performance. Therefore, it is crucial to study the relationship between the structure and performance of the carbonized ZIF-67 with the addition of CTAB. In this study, field emission scanning electron microscopy (FESEM) is employed to investigate effects of CTAB on the morphology and electrocatalytic properties of carbonized ZIF-67.

In a typical experiment, $1.43 \mathrm{~g}$ of $\mathrm{Co}\left(\mathrm{NO}_{3}\right)_{2} \cdot 6 \mathrm{H}_{2} \mathrm{O}$ and $3.24 \mathrm{~g}$ of 2-methylimidazole were dissolved in 50 $\mathrm{mL}$ methanol with or without $0.2 \mathrm{~g} / \mathrm{L} \mathrm{CTAB}$ in the solution. Then, the solution was mixed under magnetic stirring for $20 \mathrm{~min}$ and stood at $30^{\circ} \mathrm{C}$ for $24 \mathrm{~h}$. Solid product was separated by centrifugation and washed with methanol for three times, followed by vacuum drying at $40{ }^{\circ} \mathrm{C}$ for $48 \mathrm{~h}$. The as-prepared ZIF-67 above was carbonized under $\mathrm{N}_{2}$ atmosphere at $600{ }^{\circ} \mathrm{C}$ for $3 \mathrm{~h}$ with a heating rate of 2 ${ }^{\circ} \mathrm{C} / \mathrm{min}$ and naturally cooled down to room temperature. The morphology of ZIF-67 and carbonized products was characterized by FESEM (JSM-6700F, JEOL), and electrochemical tests were conducted at a rotating ring-disk electrode (RRDE-3A, ALS Co.) on a CHI760D electrochemical work station with $\mathrm{Ag} / \mathrm{AgCl}$ as reference electrode and $\mathrm{Pt}$ wire as counter electrode.

As shown in Figure1, urchin-like carbon material is obtained from carbonizing ZIF-67 without CTAB, and a plurality of elongated carbon nanotubes (CNTs) appear on the surface of main structures. The nanotubes destroy original angular structure. In contrast, with CTAB, the carbonized ZIF-67 contains a small amount of CNTs (Figure 2), and the structure is clear and angular to retain the sodalite topology of the precursor (Figure 3a). FESEM characterizations clearly indicate that the microstructure of the carbonized ZIF-67 is affected by the addition of CTAB. Electrochemical performances of the two samples are given in Figure 3b. By comparison, the carbonized ZIF-67 without CTAB has better electrochemical performance. The onset potential ( $\left.E_{\text {onset }}\right)$, half wave potential $\left(E_{1 / 2}\right)$ and limiting current density $\left(\mathrm{J}_{\mathrm{M}}\right)$ of the carbonized ZIF-67 without CTAB are $-0.067 \mathrm{~V},-0.135 \mathrm{~V}$ and $-6.39 \mathrm{~mA} \mathrm{~cm}^{-2}$, respectively, positively shifted by $43 \mathrm{mV}, 55 \mathrm{mV}$ and $0.67 \mathrm{~mA} \mathrm{~cm}{ }^{-2}$ compared with the carbonized ZIF-67 with CTAB ( $E_{\text {onset }}=-0.11 \mathrm{~V}, \mathrm{E}_{1 / 2}=-0.19 \mathrm{~V}$ and $\mathrm{J}_{\mathrm{M}}=-5.72 \mathrm{~mA} \mathrm{~cm}^{-2}$ ). This means, the formation of CNTs on the surface of carbonized ZIF-67 can serve as electron transport channels and provide more active sites for electrocatalytic reactions. 


\section{References}

[1] G. H. Zhong, D. X. Liu, J. Y. Zhang, J. Mater. Chem. A 6 (2018), p. 1887.

[2] M. Kuang, et al, Adv. Energy Mater. 7 (2017), p. 1700193.

[3] This work was partially supported by the National Natural Science Foundation of China (21776147 \& 21606140) and the International Science \& Technology Cooperation Program of China

(2014DFA60150). L. F. Dong also thanks financial support from the Malmstrom Endowment Fund at Hamline University.

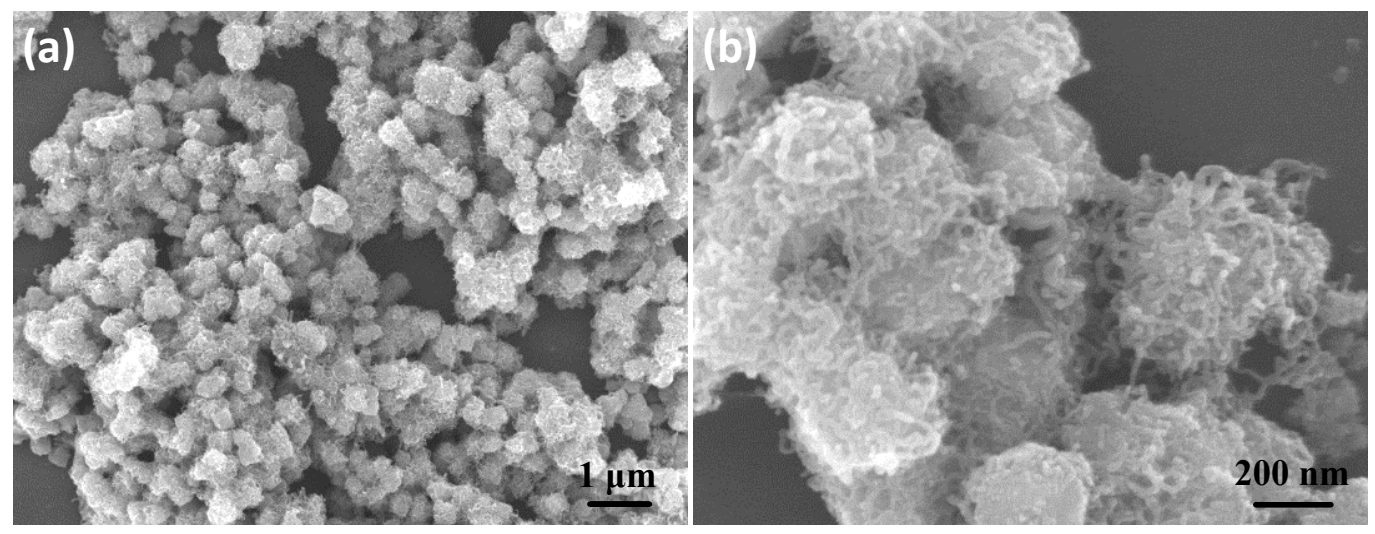

Figure 1. SEM images of carbonized ZIF-67 without CTAB.
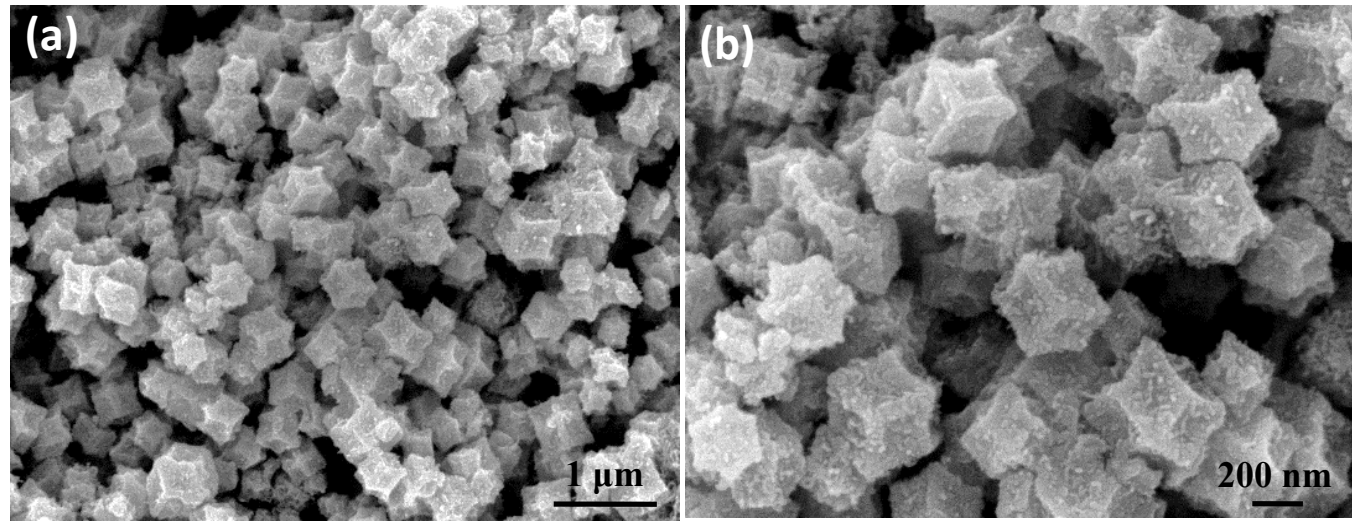

Figure 2. SEM images of carbonized ZIF-67 with the addition of CTAB.
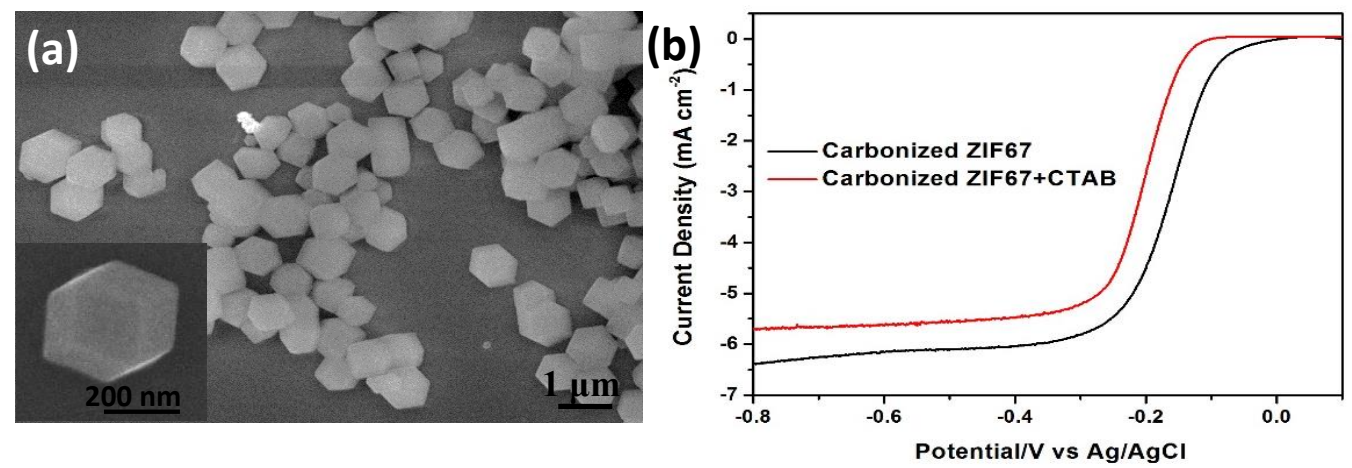

Figure 3. (a) SEM images of the ZIF-67 precursor, (b) linear sweep voltammetry (LSV) curves of carbonized ZIF-67 with/without CTAB at $1600 \mathrm{rpm}$ in $\mathrm{O}_{2}$-saturated $0.1 \mathrm{M} \mathrm{KOH}$ solution. 